\title{
Influence des tempêtes sur la mobilité des dunes tidales dans le détroit du Pas-de-Calais
}

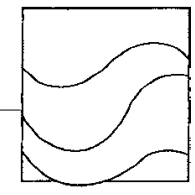

\author{
Sophie LE BOT ${ }^{a *}$, Alain TRENTESAUX ${ }^{a}$, Thierry GARLAN ${ }^{b}$, Serge BERNE ${ }^{\mathrm{c}}$, Hervé CHAMLEY ${ }^{a}$ \\ ${ }^{\text {a }}$ Laboratoire de sédimentologie et géodynamique, UMR 8577 CNRS, \\ université des sciences et technologies de Lille, bâtiment SN5, 59655 Villeneuve d'Ascq cedex, France \\ ${ }^{\mathrm{b}}$ Epshom, section géodésie-géophysique, BP 426, 29275 Brest cedex, France \\ ${ }^{\mathrm{c}}$ Ifremer, Centre de Brest, laboratoire environnements sédimentaires, DRO/GM, BP 70, \\ 29280 Plouzané cedex, France
}

Reçu le 20 octobre 1998, reçu en forme révisée le 30 août 1999, accepté le 24 octobre 1999

\begin{abstract}
Influence of storms on tidal dune mobility in the Strait of Dover. The present paper deals with dune dynamics in a zone of the Strait of Dover located in the sea lane running into the North Sea. The dunes, widespread in this $35-\mathrm{m}$ depth area, are mobile sedimentary structures (up to $40 \mathrm{~m} \cdot \mathrm{yr}^{-1}$ ) that culminate at a maximum of $22 \mathrm{~m}$ depth and endanger navigation as well as submarine man-made structures (cables, pipelines). Single- and multibeam bathymetric data, coupled with seismic data, allow us to follow dune displacements over different time scales. A net bedload parting zone has been displayed and divides the area into two parts, SE and NW. However, according to the considered time-scale, dune movements present variations in intensity and direction. Over a long-term period (decades), sedimentary dynamics fit the regional scheme of the residual tidal currents that induce transport toward the SW and the NE in the respective SE and NW parts of the studied area. Over a medium-term period (several years), meteorological data show that a high frequency of storm winds alters the residual sand transport characteristics by strengthening, slowing or reversing the effect of the tidal currents and can lead to the reversal of dune asymetry. (C) 2000 Ifremer/CNRS/IRD/Éditions scientifiques et médicales Elsevier SAS
\end{abstract}

\section{Strait of Dover / sedimentary dynamics / tidal dunes / storms}

Résumé - Cet article traite de la dynamique des dunes de sable dans une zone du détroit du Pas-de-Calais située dans le rail de navigation débouchant en mer du Nord. Les dunes de sable, répandues dans ce secteur, sont des structures sédimentaires mobiles (jusque $40 \mathrm{~m} \cdot \mathrm{an}^{-1}$ ) qui culminent jusqu'à $22 \mathrm{~m}$ de profondeur et mettent en danger la navigation ainsi que les ouvrages sous-marins (câbles, pipelines). Les données de bathymétrie monoet multifaisceaux, couplées aux données de sismique, permettent de suivre les déplacements des dunes à différentes échelles de temps. Une zone de divergence du transport sédimentaire a été mise en évidence et divise la zone en deux secteurs, SE et NW. Toutefois, selon l'échelle de temps considérée, les mouvements dunaires présentent des variations en intensité et en direction. Sur le long terme (à l'échelle de la décennie), la dynamique sédimentaire se calque sur le schéma régional des courants de marée résiduels qui induisent des transports sédimentaires vers le SW et le NE dans les secteurs respectifs SE et NW de la zone d'étude. Sur le moyen terme (à l'échelle de quelques années), les données météorologiques montrent qu'une forte fréquence des vents de tempête peut modifier les caractéristiques du transport résiduel sableux en renforçant, en ralentissant ou en inversant l'effet des courants de marée et peut conduire à l'inversion de l'asymétrie des dunes. (C) 2000 Ifremer/CNRS/IRD/Éditions scientifiques et médicales Elsevier SAS

détroit du Pas-de-Calais / dynamique sédimentaire / dunes tidales / tempêtes

\footnotetext{
* Correspondance et tirés à part: sophie.le-bot@univ-lille1.fr
} 


\section{INTRODUCTION}

\subsection{Généralités}

Les dunes et les bancs sableux sous-marins, fréquents sur les plates-formes continentales, sont connus depuis les descriptions de Hider [25], Lüders [31] et Van Veen [46]. Les connaissances sur ces corps sédimentaires ont beaucoup progressé grâce au développement des méthodes acoustiques telles que le sondage bathymétrique, l'imagerie par sonar à balayage latéral $[2,3,5,40]$ et la sismique à haute et très haute résolution $[6,26]$. Les bancs sableux constituent de grandes structures longues de plusieurs dizaines de kilomètres et larges de quelques kilomètres; ils sont légèrement obliques par rapport aux directions principales des courants de marée. Les dunes sableuses, de dimensions plus faibles (quelques centaines de mètres à plusieurs kilomètres de long, quelques mètres à un kilomètre de large), façonnent le sommet des bancs sableux et en constituent la structure [8] ou se forment indépendamment et se rencontrent isolées. Elles sont quasiment perpendiculaires au grand axe de l'ellipse des courants de marée. Contrairement aux bancs sableux qui sont réputés très peu mobiles voire stables $[11,26,43]$, les dunes de sable isolées sont susceptibles de se déplacer très rapidement. En mer du Nord, Stride [41] a enregistré des déplacements atteignant $150 \mathrm{~m} \cdot \mathrm{an}^{-1}$, De Moor [16] plus de $150 \mathrm{~m} \cdot \mathrm{an}^{-1}$ et Berné et al. [7] $80 \mathrm{~m} \cdot \mathrm{an}^{-1}$. Elles constituent de ce fait une source potentielle de risques pour l'installation d'ouvrages en mer (câbles, pipelines...) et la navigation dans les secteurs peu profonds. L'étude de la mobilité des corps sableux sous-marins fait l'objet de diverses actions et collaborations internationales. En France, la surveillance des zones navigables est assurée par le service hydrographique et océanographique de la marine (Shom) qui réalise un levé hydrographique détaillé des zones sensibles tous les deux à trois ans. Les données recueillies permettent de préciser les différents mécanismes qui contrôlent la direction et l'intensité des transits sédimentaires. Parmi les secteurs surveillés par le Shom, le Pas-de-Calais est une zone particulièrement sensible.

\subsection{Le Pas-de-Calais}

Situé sur la plate-forme épicontinentale du NW de
l'Europe, ce détroit n'excède pas $50 \mathrm{~m}$ de profondeur (figure 1). Depuis sa submersion au stade Boréal (Holocène inférieur), il relie les bassins de la Manche et de la mer du Nord [14, 27]. Les fonds marins sont tapissés de nombreuses accumulations sédimentaires représentées par des grandes dunes et des bancs sableux qui culminent généralement à moins de $30 \mathrm{~m}$ de profondeur $(6 \mathrm{~m}$ pour le South Falls, $5 \mathrm{~m}$ pour le Sandettié). Ces corps sédimentaires se sont mis en place lors de la transgression holocène $[18,27]$ à partir de stocks sableux issus principalement des décharges du système fluviatile Rhin-Meuse et de la Tamise durant les stades glaciaires et interglaciaires du Pléistocène [26, 49].

Le Pas-de-Calais est le siège du trafic maritime le plus intense au monde. Environ 300 navires y transitent tous les jours, en particulier dans les deux rails de navigation NE-SW qui couvrent la majeure partie de sa superficie. La densité du réseau d'ouvrages en mer est également élevée. Compte tenu de ces caractéristiques, le Pas-de-Calais constitue un domaine clef pour lequel la sécurité de la navigation et la préservation des ouvrages sont conditionnées par une connaissance précise de la dynamique sédimentaire et des facteurs responsables de la mobilité des corps sableux.

\subsection{Objectif de l'étude}

En Manche occidentale et dans le sud de la mer du Nord, les courants de marée sont les principaux agents responsables du transport sédimentaire et leurs caractéristiques sont bien connues. Dans le Pas-de-Calais, la formation et la mobilité des grandes dunes sont principalement liées à ce phénomène cyclique. Toutefois, des modifications sporadiques des modalités de la migration de ces corps sableux tidaux suggèrent l'influence d'autres facteurs. Afin de les caractériser, le déplacement des dunes a été étudié selon plusieurs échelles de temps : décennale, pluriannuelle et événementielle.

\section{ZONE D’ÉTUDE}

Cette étude est focalisée sur une zone du Pas-deCalais située à mi-distance entre Calais et Douvres, sur la voie de navigation débouchant en mer du Nord (figure 1). Il s'agit d'une des zones sensibles 
surveillées par le Shom, qui l'a nommée «zone F ». Elle est située au centre du Pas-de-Calais à proximité de la partie la plus étroite du détroit (environ $30 \mathrm{~km}$ ) et des plus faibles profondeurs. Cette configuration implique une exacerbation des facteurs hydrodynamiques (marées et houles) selon une direction NESW.

La zone $\mathrm{F}$ se trouve à la convergence des bancs sableux du South Falls et du Sandettié. Les corps sédimentaires présents correspondent, selon la clas- sification de Berné et al. [7] et Ashley [1], à des grandes dunes dont la base se trouve située vers $35 \mathrm{~m}$ de profondeur (D) et le sommet à $22 \mathrm{~m}$ sous le niveau des plus basses mers. Elles sont pour la plupart rectilignes mais les plus grandes tendent à être sinueuses. La longueur des crêtes des dunes varie entre $750 \mathrm{~m}$ et $3 \mathrm{~km}$. Ces dunes s'organisent en deux ensembles morphologiquement contrastés :

a) dans le secteur $\mathrm{SE}$, les dunes présentent des espacements (L) moyens de $750 \mathrm{~m}$ et des hauteurs (H)

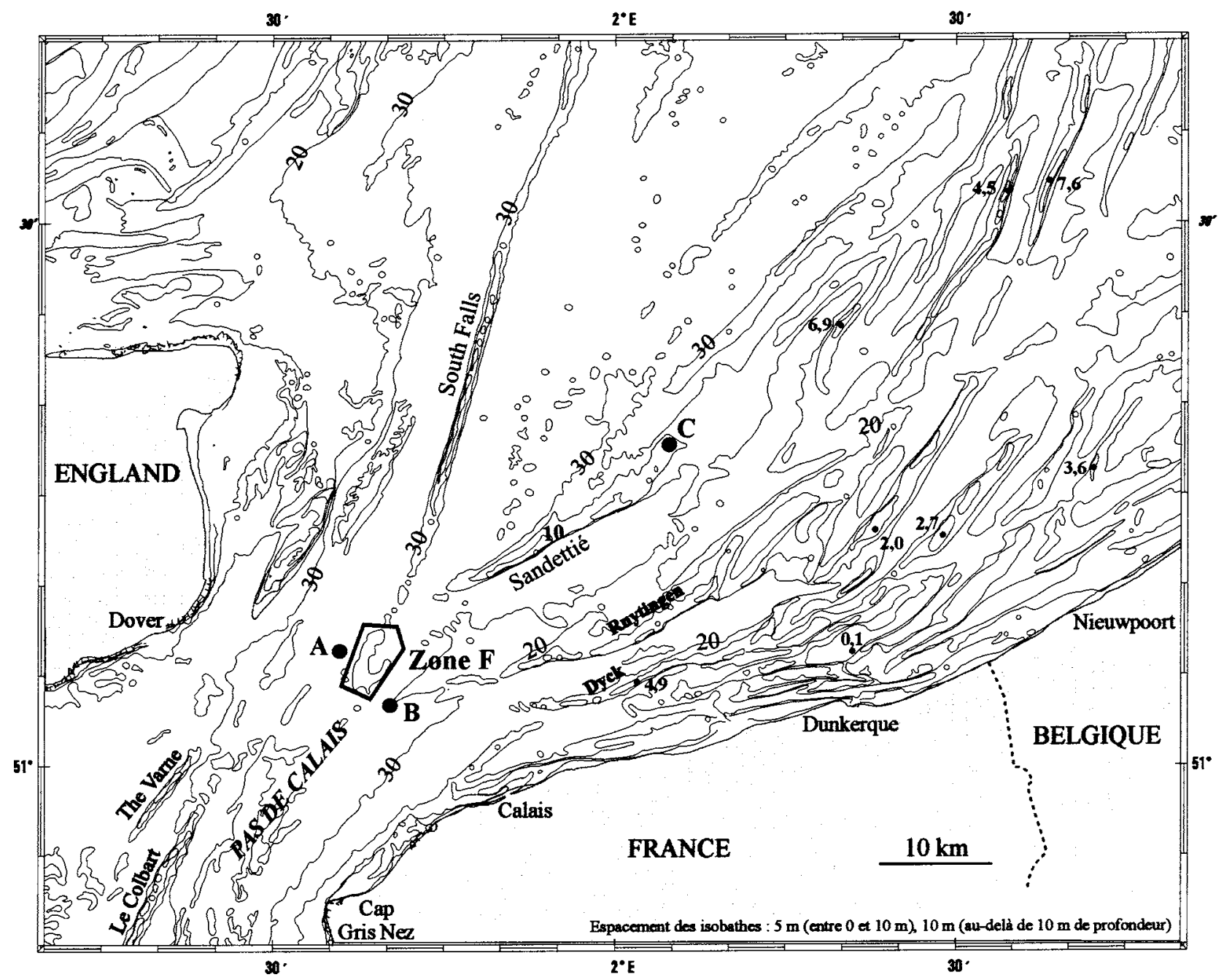

Figure 1. Carte bathymétrique du sud de la mer du Nord et du détroit du Pas-de-Calais (extrait de la carte nº735, Shom). Localisation de la zone $\mathrm{F}$ et des sites de mesure de courant $\mathrm{A}, \mathrm{B}$ et $\mathrm{C}$.

Figure 1. Bathymetric chart of the southern North Sea and the Dover Strait (extract from chart 6735, S.H.O.M.). Location of study area (zone F) and current measurement sites A, B and C. 
moyennes de $10 \mathrm{~m}$ (jusqu'à $13 \mathrm{~m}$, au maximum); elles s'allongent avec une direction $\mathrm{N} 120^{\circ}$ et sont asymétriques vers le SW, sauf les plus petites qui sont parfois symétriques; le fond est compris entre 34 et $39 \mathrm{~m}$;

b) dans le secteur NW, les dunes sont en moyenne espacées de $350 \mathrm{~m}$ et hautes de $7 \mathrm{~m}$; leur allongement est orienté $\mathrm{N} 150^{\circ}$; elles sont majoritairement asymétriques vers le NE, certaines sont symétriques ; le fond est situé entre 31 et $33 \mathrm{~m}$.

La zone de contact entre ces deux entités est orientée $\mathrm{N} 45^{\circ}$; elle est marquée par la présence de quelques portions de dunes symétriques. Trois dunes traversent ces deux secteurs et présentent les variations morphologiques propres à chacun d'eux.

La formule de Flemming [19], $\mathrm{H}=0,0677 \cdot \mathrm{L}^{0,8098}$, a été établie à partir de 1491 observations sur des corps sédimentaires transverses aux courants principaux. Elle donne des hauteurs de 7,5 et $14 \mathrm{~m}$ pour des espacements respectifs de 350 et $750 \mathrm{~m}$, valeurs légèrement supérieures à celles mesurées. Flemming [19] suggère que l'équilibre entre la morphologie des dunes et les conditions hydrodynamiques est plus difficile à atteindre lorsque les dunes sont de plus grande taille. De même, Perillo et Lüdwick [33] montrent que les grandes dunes ont des hauteurs inférieures aux valeurs calculées, car elles sont fréquemment appauvries en sédiment. Les formules de Yalin [48], $\mathrm{H}=0,167 \cdot \mathrm{D}$ et $\mathrm{L}=6 \cdot \mathrm{D}$, résultent de la combinaison de la théorie et des observations. Elles donnent les valeurs maximales des hauteurs et espacements des dunes en fonction de la profondeur [48]. Pour des profondeurs respectives de 32 et $36 \mathrm{~m}$ (moyennes des secteurs NW et SE), ces formules conduisent à des hauteurs de 5,3 et $6 \mathrm{~m}$ et des espacements de 192 et $216 \mathrm{~m}$, valeurs faibles par rapport aux observations. Dalrymple et Rhodes [15] suggèrent que, dans le cas de superposition de rides et de petites dunes à la surface des grandes, les relations entre $\mathrm{H}, \mathrm{L}$ et $\mathrm{D}$ soient plus complexes que celles de Yalin [48]. Dans l'estuaire de la Gironde, Berné et al. [9] observent que la hauteur des dunes augmente plus rapidement que ce qui est prédit par Yalin [48] lorsque la profondeur d'eau dépasse $30 \mathrm{~m}$; la différence est attribuée à certains paramètres comme la granulométrie du sédiment et la vitesse du courant, variables à l'échelle d'une dune, et qui ne sont pas pris en compte dans les formules.

\section{MÉTHODES}

Les mouvements des dunes sableuses ont été suivis grâce aux données de bathymétrie mono- et multifaisceaux recueillies par le Shom. La réalisation de cartes de position des crêtes de dunes a permis de quantifier les déplacements au cours du temps [24]. L'architecture interne des dunes a été révélée par des données de sismique réflexion à très haute résolution (THR) (sondeur de sédiment $3,5 \mathrm{kHz}$; mission Sepia II, Insu/CNRS, juin 1996). Les profils sismiques, qui pénètrent la totalité des corps dunaires $(10$ à $13 \mathrm{~m})$, ont conduit à reconnaître leur géométrie interne et à définir des microséquences délimitées par trois types de discontinuités [8]: des discontinuités de premier ordre sub-horizontales interprétées comme des surfaces d'érosion, des discontinuités de second ordre obliques résultant de la combinaison épisodique de la marée et des houles, et des discontinuités de troisième ordre, plus obliques, attribuées au phénomène de réactivation par le courant subordonné [10]. Des analyses granulométriques ont été effectuées à partir de sédiments de surface prélevés à la benne Shipeck et ont mis en évidence deux ensembles granulométriques majeurs: des cailloutis et graviers constituant une «semelle» sédimentaire et des sables moyens de $0,3 \mathrm{~mm}$ de diamètre qui composent les dunes recouvrant partiellement cette «semelle». Des mesures de courantologie (courantomètres Suber et Doppler) et de vents, collectées respectivement par le Shom et Météo France, ont permis de caractériser les agents hydrodynamiques susceptibles de jouer un rôle moteur pour la dynamique sédimentaire.

\section{INFLUENCE DES MARÉES}

\subsection{Mobilisation du sédiment par les courants de marée}

Le Pas-de-Calais est soumis à un régime macrotidal semi-diurne, dont le marnage atteint $6 \mathrm{~m}$ en viveseaux et 3,5 m en mortes-eaux. Les courants de marée présentent un caractère alternatif marqué du fait de la configuration du détroit. Dans la partie centrale, où 
est située la zone F, le jusant dirigé vers le SW domine sur le flot orienté vers le NE [36]. Toutefois, en bordure des littoraux français et anglais, le flot est la phase de courant dominante [36]. Les bancs du South Falls et du Sandettié déterminent un vaste entonnoir [47] qui canalise au NE le courant de jusant. Il en résulte une accélération de ce courant qui est maximal au NE de la zone F.

Des mesures de courant de marée ont été réalisées à l'aide de courantomètres Suber [35] en deux points situés à l'ouest (point $\mathrm{A}$ ) et au sud (point B) de la zone F (figure 1). Sur les deux sites de mesure, le jusant et le flot portent respectivement vers $\mathrm{N} 35^{\circ}-$ $\mathrm{N} 45^{\circ}$ et $\mathrm{N} 210^{\circ}-\mathrm{N} 220^{\circ}$. Les vitesses sont comprises entre $0,10 \mathrm{~m} \cdot \mathrm{s}^{-1}$ et $1,85 \mathrm{~m} \cdot \mathrm{s}^{-1}$. La durée du jusant excède celle du flot d'1 $h$ environ. Les vitesses des pointes de jusant sont supérieures à celles des pointes de flot de $0,2 \mathrm{~m} \cdot \mathrm{s}^{-1}$ en mortes-eaux et de $0,4 \mathrm{~m} \cdot \mathrm{s}^{-1}$ en vives-eaux. Le courant de jusant atteint $1 \mathrm{~m} \cdot \mathrm{s}^{-1}$ en mortes-eaux et $1,85 \mathrm{~m} \cdot \mathrm{s}^{-1}$ en vives-eaux (point $\mathrm{B}$ ). Les vitesses au point $\mathrm{B}$ sont supérieures à celles mesurées au point A d'environ $0,2 \mathrm{~m} \cdot \mathrm{s}^{-1}$ en morteseaux et $0,3 \mathrm{~m} \cdot \mathrm{s}^{-1}$ en vives-eaux.

Selon Dyer [17] le seuil de mobilisation d'un sédiment de $0,3 \mathrm{~mm}$ de diamètre, cas du sable de nos dunes, correspond à une vitesse critique de frottement sur le fond d'environ $1,5 \mathrm{~cm} \cdot \mathrm{s}^{-1}$. On admet un profil de vitesse logarithmique sur l'ensemble de la tranche d'eau décrit par l'équation de von Karman-Prandtl [17] :

$\mathrm{U}_{\mathrm{z}}=\mathrm{U} * / 0,4 \cdot \ln \left(\mathrm{Z} / \mathrm{Z}_{0}\right)$

où $\mathrm{U}_{\mathrm{z}}$ est la vitesse du courant à la hauteur $\mathrm{Z}$ au-dessus du fond, $U *$ la vitesse de frottement sur le fond, et $Z_{0}$ le paramètre de rugosité qui est directement relié au diamètre $\mathrm{d}$ du sédiment par la relation $\mathrm{Z}_{0}=\mathrm{k}_{\mathrm{s}}^{\prime} / 30$ avec $\mathrm{k}_{\mathrm{s}}=3 \cdot \mathrm{d}_{90}[34]$.

On montre qu'une vitesse de friction sur le fond de $1,5 \mathrm{~cm} \cdot \mathrm{s}^{-1}$ correspond à une vitesse de courant en surface $\left(\mathrm{U}_{\mathrm{z}}=\mathrm{U}_{35}\right)$ de $0,5 \mathrm{~m} \cdot \mathrm{s}^{-1}$. En vives-eaux, une telle vitesse est dépassée durant plus de $5 \mathrm{~h}$ lors de la phase de jusant et plus de $4 \mathrm{~h}$ lors de la phase de flot. En mortes-eaux, elle est dépassée pendant plus de $3 \mathrm{~h}$ et $4 \mathrm{~h}$ respectivement. En conséquence, le sédiment constituant les dunes de la zone F est susceptible d'être mobilisé à chaque phase du courant de marée, et cela quel qu'en soit le coefficient.

\subsection{Mouvements des dunes sous l'effet des courants de marée. Exemple de la période 1985-1995 (figure 2a)}

Durant les dix années concernées par notre étude, les dunes du secteur SE ont migré activement vers le SW (10 $\mathrm{m} \cdot \mathrm{an}^{-1}$, en moyenne) alors que celles du secteur NW se sont déplacées vers le NE mais moins rapidement ( $3 \mathrm{~m} \cdot \mathrm{an}^{-1}$, en moyenne). Ces tendances sont confirmées par les données de sismique THR pour la totalité de la période de construction des dunes. En effet, sur les profils sismiques (figure 3), les réflecteurs internes de type 2 (discontinuités de second ordre), indicateurs du sens du transit sédimentaire, montrent essentiellement des pendages vers le SW pour les dunes du secteur SE (figure 3-P1), alors que pour le secteur NW ils sont en majorité orientés vers le NE (figure 3P2).

Il résulte de ces observations qu'à l'échelle de la décennie les mouvements des dunes au sein de la zone F sont conformes au schéma des courants tidaux. Les dunes isolées du secteur SE subissent l'influence directe du jusant dominant orienté vers le SW. En revanche, les dunes du secteur NW, qui constituent l'extrémité méridionale du South Falls, sont relativement protégées de l'action du jusant et semblent subir préférentiellement des circulations propres aux bancs sableux [29] en direction du NE. La séparation de ces influences suit une ligne de direction $\mathrm{N} 45^{\circ}$ (figure 2a), qui est celle du grand axe de l'ellipse des courants tidaux dans le Pas-de-Calais. Cette divergence de la direction du transport sédimentaire résiduel coïncide avec la frontière morphologique décrite précédemment (cf. paragraphe 2). En conséquence, à l'échelle de toute la zone, le transport sédimentaire résiduel montre une tendance à la rotation horaire. Les trois plus grandes dunes qui traversent la frontière confirment ce résultat: leur portion NW migre vers le NE et celle située dans le secteur SE se déplace vers le SW. Smith $[37,38]$ a déjà mis en évidence une telle tendance pour les dunes de la terminaison SW du banc du South Falls, situées à $8 \mathrm{~km}$ au NE de la zone F. D'autres auteurs ont observé un phénomène comparable pour des dunes isolées du sud de la mer du Nord [12, 26, 32, 39]. 

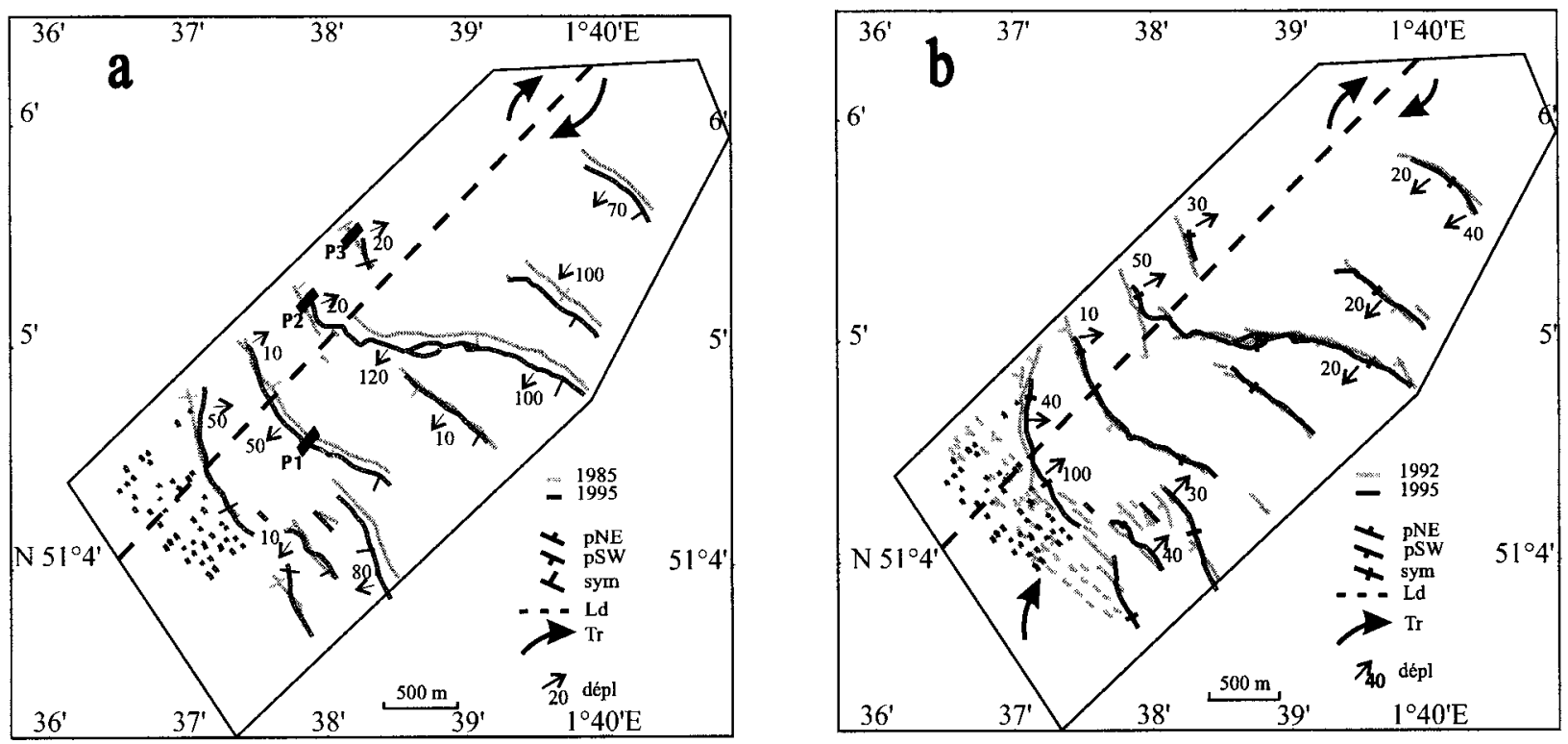

Figure 2. Migration des crêtes de dunes de la zone F, a : entre 1985 et 1995, b : entre 1992 et 1995 (d'après Herman [24]). Rectangles noirs : profils sismiques; pNE, pSW : dune à polarité orientée vers le nord-est, le sud-ouest ; sym : dune symétrique ; Ld : ligne de divergence du transport résiduel; $\operatorname{Tr}$ : direction du transport résiduel ; dépl : déplacement des crêtes en mètres.

Figure 2. Dune crest migration in area F, a: between 1985 and 1995 and b: between 1992 and 1995 (after Herman [24]). Black boxes: selected seismic profiles; pNE, pSW: northeast-, southwest-facing dune; sym: symetric dune; Ld: net bedload parting line; Tr: residual transport direction; dépl: dune displacement in metres.

\section{INFLUENCE DES TEMPÊTES}

Si les vagues et les houles engendrées par les vents ont une influence connue et souvent marquée sur les domaines côtiers les moins profonds [4, 44], seules celles résultant de tempêtes peuvent modifier sensiblement les fonds plus importants du Pas-de-Calais. Selon Grochowsky et al. [20], les houles ont un effet négligeable sur le transport sédimentaire dans cette région, contrairement aux vents. Les vents dominants $[13,30]$ de secteur SW, sont à la fois les plus fréquents $(14 \%)$ et les plus puissants (vitesses souvent supérieures à $7 \mathrm{~m} \cdot \mathrm{s}^{-1}$ ). Toutefois, ceux de secteur NE sont dominants au printemps (fréquence $10 \%$, vitesse maximale $5 \mathrm{~m} \cdot \mathrm{s}^{-1}$ ).

\subsection{Mesures lors d'une période de tempête. Exemple du mois de septembre 1995 (figure 4)}

Une tempête d'origine cyclonique (cyclone Isis) a traversé le sud de la mer du Nord en septembre 1995. La dépression à l'origine de cette tempête (figure $4 a$ ) s'est amorcée le $1^{\text {er }}$ septembre pour atteindre son paroxysme le 8 septembre ( 980 mbar) et se résorber le 18 septembre. Au cours de cette période, les vents sont puissants et montrent d'importantes variations de direction (figure $4 b$ ).

Du 25 août au 2 septembre, les vents soufflent du secteur NW, passant à N-NE avec des vitesses faiblissant de 15 à $6 \mathrm{~m} \cdot \mathrm{s}^{-1}$ en moyenne.

Le 2 septembre, l'installation de la dépression est soulignée par un rapide changement de la direction des vents : en quelques heures, ils passent progressivement de secteur NW à secteur S-SW. Du 2 au 18 septembre, les vents sont plus modérés $\left(5 \mathrm{~m} \cdot \mathrm{s}^{-1}\right.$ en moyenne) et s'établissent globalement depuis le S-SW, passant progressivement au secteur S. Toutefois lors $\mathrm{du}$ passage du centre dépressionnaire, les vents sont forts et changent rapidement de direction : le 7 septembre, ils soufflent du SE et atteignent $12 \mathrm{~m} \cdot \mathrm{s}^{-1}$; le 8 , ils proviennent de l'ouest et soufflent à $15 \mathrm{~m} \cdot \mathrm{s}^{-1}$., Du 16 au 18 septembre, le comblement de la dépres- 
sion s'accompagne de vents qui tournent du secteur SE jusqu'au secteur E et atteignent $11 \mathrm{~m} \cdot \mathrm{s}^{-1}$.

Du 19 au 23 septembre, les vents soufflent des secteurs NE à N-NE, excepté durant quelques heures les 21 et 22 où ils proviennent de l'W-NW. Particulièrement puissants le $19\left(20 \mathrm{~m} \cdot \mathrm{s}^{-1}\right)$, ils faiblissent rapidement $\left(6 \mathrm{~m} \cdot \mathrm{s}^{-1}\right.$ de moyenne).
Parallèlement, la courantométrie Doppler a été effectuée sur l'ensemble de la tranche d'eau au nord du banc du Sandettié, à une trentaine de kilomètres de la zone $\mathrm{F}$ (site $\mathrm{C}$, figure 1). Les caractéristiques de la marée y sont identiques à celles de la zone $\mathrm{F}[36]$ et le jusant, dirigé vers le SW, est également la phase de courant dominante. Toutefois, les pointes de jusant et de flot sont inférieures de $0,8 \mathrm{~m} \cdot \mathrm{s}^{-1}$ et atteignent
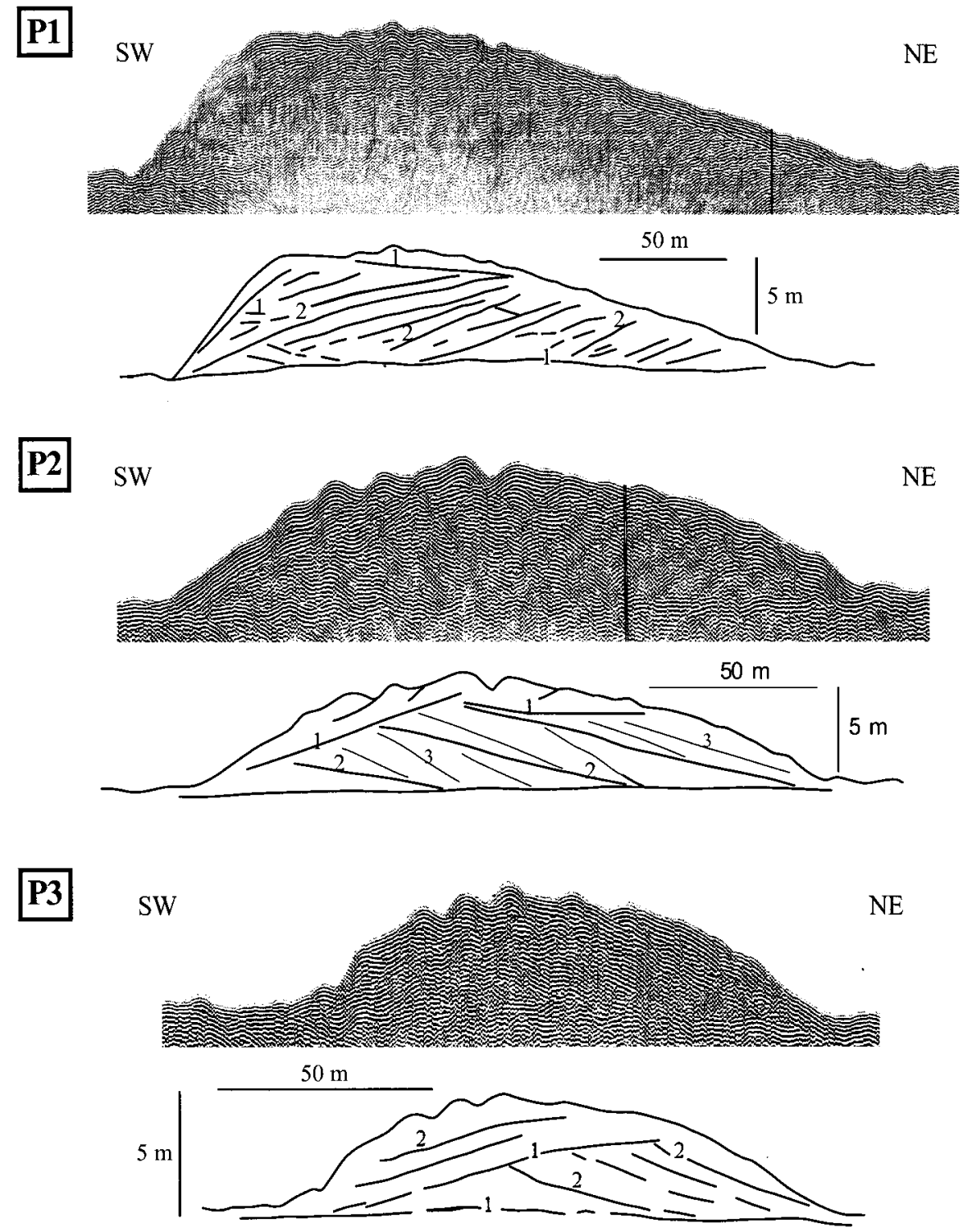

Figure 3. Sections sismiques P1, P2 et $\mathrm{P} 3$ transversales aux dunes et leurs interprétations (localisation des sections sur la figure 2a). 1, 2 et 3 : réflecteur de premier, second et troisième ordre.

Figure 3. P1, P2 and P3 seismic sections transverse to dunes and their interpretations (section location on figure 2a). 1, 2 and 3: first, second and third order reflector. 

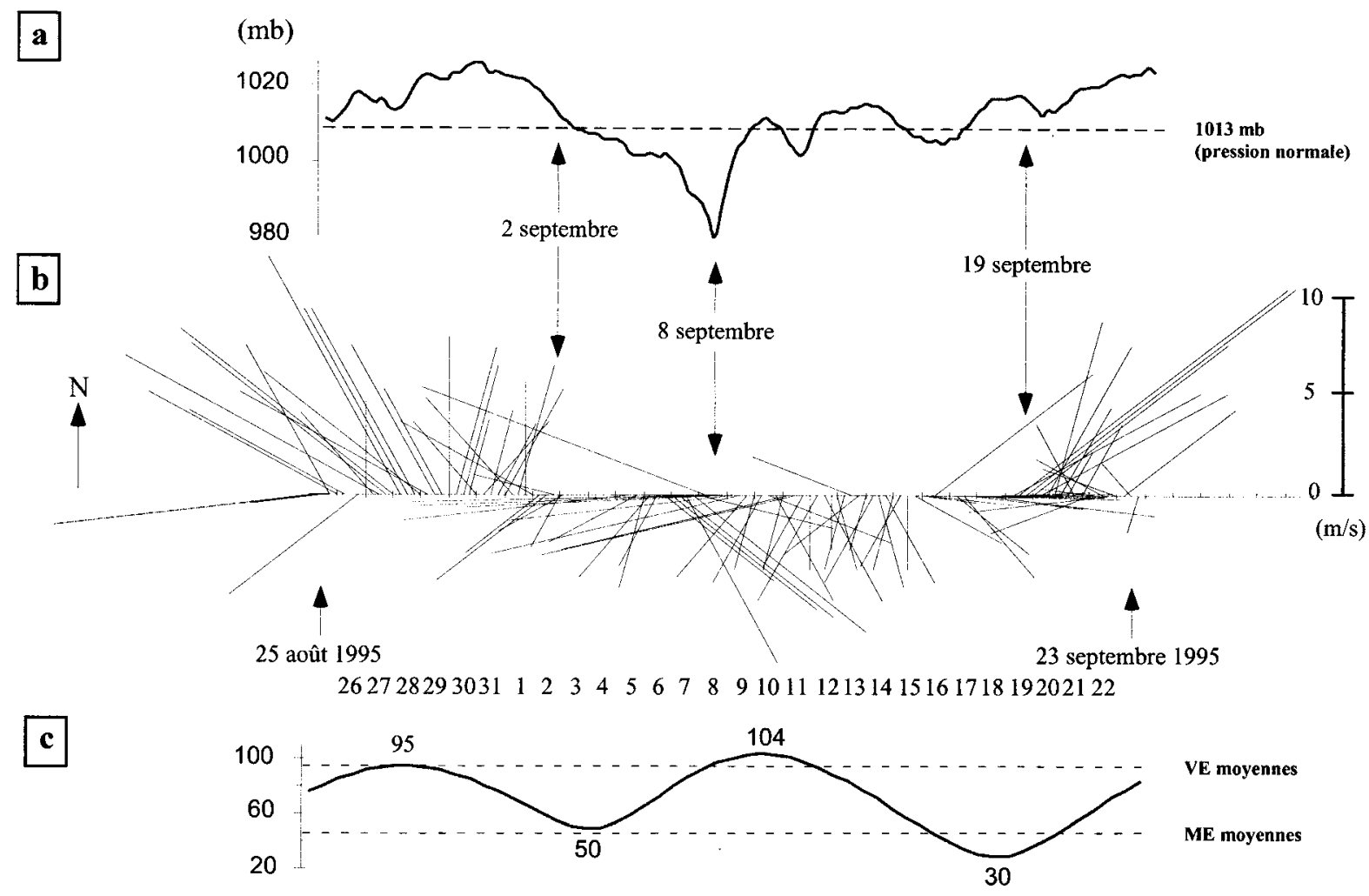

$\mathbf{d}$

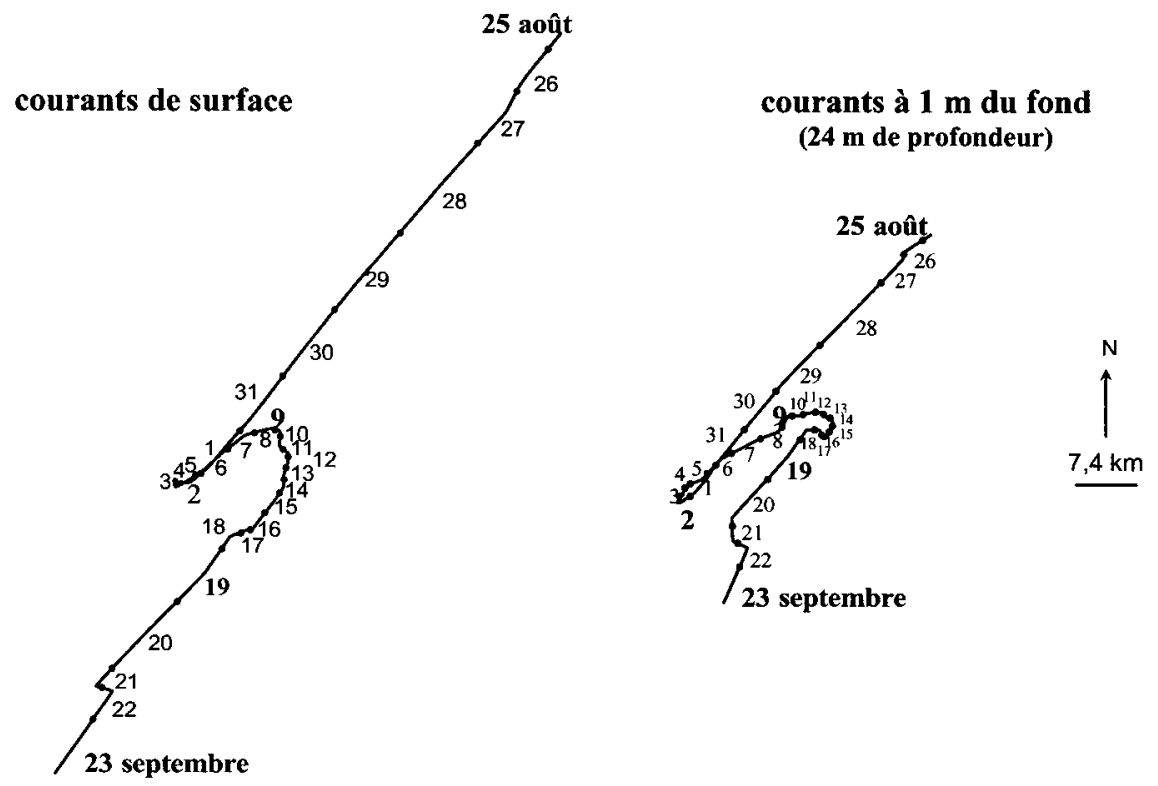

Figure 4. Paramètres hydrodynamiques et météorologiques enregistrés lors de la tempête de septembre 1995. a : pression atmosphérique, $\mathrm{b}$ : vents au sémaphore de Dunkerque (représentation eulérienne), c : coefficients de marée, et, $\mathrm{d}$ : courants résiduels au site $\mathrm{C}$ (hodographe intégré, représentation lagrangienne).

Figure 4. Hydrodynamic and meteorological parameters recorded during the storm of september 1995. a: atmospheric pressure, b: winds at Dunkerque signal station (eulerian representation), c: tidal coefficients, d: residual currents at site C (integrated hodograph, lagrangian representation). 
respectivement 1,03 et $0,93 \mathrm{~m} \cdot \mathrm{s}^{-1}$ en vives-eaux moyennes. Durant la tempête, le courant résiduel, intégré sur la journée, ne montre pas de signature tidale exclusive à effet de jusant dominant (figure 4d): il subit l'influence des forts vents de tempête.

Du 25 août au 2 septembre, le courant résiduel est dirigé vers le S-SW et induit un déplacement important de la masse d'eau dans cette direction $\left(10 \mathrm{~km} \cdot \mathrm{j}^{-1}\right.$ en moyenne). L'accélération des courants résiduels vers le S-SW s'explique par une augmentation de la puissance du jusant (pointes maximales à $1,15 \mathrm{~m} \cdot \mathrm{s}^{-1}$ ) et une diminution de celle du flot (pointes maximales à $\left.0,75 \mathrm{~m} \cdot \mathrm{s}^{-1}\right)$. Les vents de secteur $\mathrm{NW}$ à $\mathrm{N}-\mathrm{NE}$ renforcent l'action du jusant et amoindrissent celle du flot, d'autant plus qu'ils sont puissants et leurs sont parallèles. En particulier le 28 août, la combinaison d'un maximum de vives-eaux moyennes (figure $4 c$ ) et de forts vents de N-NW $\left(14 \mathrm{~m} \cdot \mathrm{s}^{-1}\right)$ engendre un courant résiduel vers le $\mathrm{S}-\mathrm{SW}$ (14 km de déplacement cumulé).

Du 2 au 18 septembre, le courant résiduel présente des variations importantes. La masse d'eau montre un faible déplacement cumulé de 3,5 km vers l'E-SE. À partir du 2 et jusqu'au 8 septembre, le courant résiduel s'inverse radicalement en direction NE. En cette période de mortes-eaux moyennes (figure 4c), les courants de marée sont plus faibles et les vents de SW, quoique modérés, suffisent à renforcer le flot pour le rendre supérieur au jusant. Dès le 2 septembre, les pointes de flot sont supérieures de $15 \mathrm{~cm} \cdot \mathrm{s}^{-1}$ à celles du jusant. Le 8 septembre, les forts vents d'ouest dévient la masse d'eau vers l'est. À partir du 9 et jusqu'au 18 septembre, le courant résiduel subit une nouvelle inversion et retrouve progressivement une direction $\mathrm{S}-\mathrm{SW}$, mais le déplacement de la masse d'eau est faible (quelques kilomètres par jour). Le 9 septembre, les vents de SW n'ont plus autant d'influence sur les forts courants de marée de vives-eaux. Le courant résiduel retrouve ses caractéristiques tidales à jusant dominant. Il demeure toutefois faible jusqu'au 18 septembre sous l'effet des vents de SW qui ralentissent le jusant. Les pointes de jusant dépassent celles du flot de moins de $10 \mathrm{~cm} \cdot \mathrm{s}^{-1}$.

Du 19 au 23 septembre, le courant résiduel, orienté vers le S-SW, est important. Les forts vents de $\mathrm{N}-\mathrm{NE}$ renforcent le jusant. Les 21 et 22 septembre, les faibles vents d'W-NW dévient légèrement les courants de mortes-eaux vers l'E-SE.

À $1 \mathrm{~m}$ au-dessus du fond (figure $4 d$ ), les courants résiduel et instantané montrent les mêmes perturbations qu'en surface. Toutefois, l'inversion y est plus prononcée : elle perdure jusqu'au 13 septembre, soit $4 \mathrm{j}$ de plus qu'en surface. Le déplacement cumulé de la masse d'eau vers le NE entre le 2 et le 8 septembre est de $22 \mathrm{~km}$ sur le fond contre $15 \mathrm{~km}$ en surface. Observant les effets d'une tempête, Heathershaw [23] note une modification persistante de la direction du courant résiduel sur le fond jusqu'à $20 \mathrm{j}$ après l'événement. Durant l'inversion, les pointes de flot (55 $\mathrm{cm} \cdot \mathrm{s}^{-1}$ en moyenne) sont supérieures de 10 $\mathrm{cm} \cdot \mathrm{s}^{-1}$ à celles du jusant. Ces fortes vitesses témoignent de l'impact important d'une telle inversion sur le transport sédimentaire à court terme.

En résumé, lors de la tempête de septembre 1995, l'influence du vent permet d'expliquer les perturbations du courant observées sur l'ensemble de la tranche d'eau. Les vents de NE renforcent le jusant. Ceux de SW, au contraire, le ralentissent et peuvent même conduire à une inversion de la prédominance des phases de courant : le flot devient la phase dominante. Une telle inversion est favorisée par la combinaison de forts vents de SW et de conditions de mortes-eaux. La corrélation entre les caractéristiques $\mathrm{du}$ vent et celles du courant marin est directe et quasiment synchrone. Toutefois, l'inversion persiste plus longtemps sur le fond induisant de fortes modifications du transport sédimentaire.

Ainsi, dans les milieux peu profonds, les vents sont en mesure de modifier fortement la circulation liée aux courants de marée sur l'intégralité de la colonne d'eau et affectent le transport sédimentaire.

\subsection{Déplacements sous les effets combinés de la marée et des tempêtes. Exemple des périodes 1985-1992 et 1992-1995 (figure 2b)}

De 1992 à 1995, le déplacement des crêtes des dunes du secteur NW s'effectue vers le NE de manière très active (jusqu'à $17 \mathrm{~m} \cdot \mathrm{an}^{-1}$ de migration totale) (figure $2 b$ ). Dans le secteur SE, la migration des dunes est globalement plus faible et s'effectue vers le SW pour les dunes septentrionales $\left(8 \mathrm{~m} \cdot \mathrm{an}^{-1}\right.$ en moyenne), 


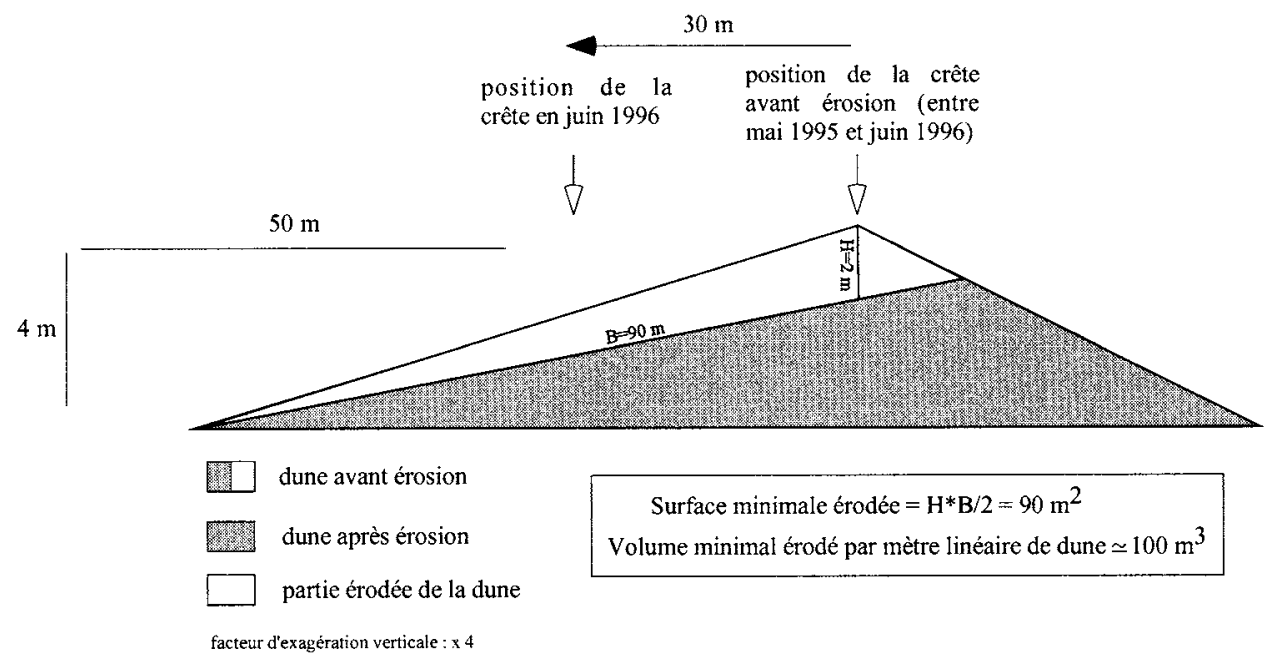

Figure 5. Modification morphologique de la dune P3 liée à l'érosion majeure. Schéma établi à partir des données de sismique de la figure 3 (les pendages des flancs de la dune correspondent à ceux mesurés en juin 1996).

Figure 5. Morphological modifications of the P3 dune in relation with the major erosion. Figure established from the seismic data presented in figure 3 (dune slopes correspond to those measured in June 1996).

vers le NE pour les dunes méridionales $\left(13 \mathrm{~m} \cdot \mathrm{an}^{-1} \mathrm{en}\right.$ moyenne et jusqu'à $\left.40 \mathrm{~m} \cdot \mathrm{an}^{-1}\right)$. Par soustraction, entre 1985 et 1992, les crêtes de dunes se sont toutes déplacées vers le SW à l'exception de la dune la plus occidentale de la zone F. L'organisation de la dynamique sédimentaire au cours de ces périodes est différente de celle observée entre 1985 et 1995, principalement en ce qui concerne l'importance des déplacements mais aussi leur direction. Il en résulte que les facteurs tidaux responsables de la migration des dunes à long terme (décennie) n'expliquent pas celle observée à moyen terme (quelques années). Les courants de marée, qui agissent en permanence, ne sont donc pas seuls à contrôler la mobilité des dunes de sable. Les changements d'intensité et de direction des mouvements dunaires sur le moyen terme, comparativement au long terme, semblent résulter de l'action d'agents hydrodynamiques, exercée vers le SW entre 1985 et 1992, et vers le NE entre 1992 et 1995.

L'étude des profils sismiques THR met en évidence des irrégularités dans la construction des dunes. Des réflecteurs sub-horizontaux de premier ordre apparaissent sporadiquement dans les corps dunaires (figure 3 P1, P3). Ces discontinuités, qui tronquent les unités sous-jacentes, correspondent à des surfaces d'érosion. Dans le cas de la dune P1, leur extension est mineure. Par contre, l'érosion affecte toute la partie sommitale de la dune P3 (figure 3, P3) et concerne, au minimum, un volume estimé à $100 \mathrm{~m}^{3}$ par mètre linéaire de dune (figure 5). Elle s'accompagne d'une reconstruction de la dune dans la direction opposée. L'asymétrie des courants tidaux semi-diurnes ne peut être seule invoquée pour expliquer ce phénomène, car le temps de réponse de la morphologie des dunes de cette taille est bien supérieur à la durée d'un cycle tidal semi-diurne. Seuls des processus de haute énergie comme les tempêtes sont en mesure de provoquer une inversion majeure du courant capable d'éroder instantanément de tels volumes sableux et de modifier le transport sédimentaire jusqu'à renverser le sens de progradation des dunes. D'une migration dirigée vers le NE entre 1985 et 1995, la dune du profil P3, observée en juin 1996, met en évidence une inversion suivie d'une reconstruction active en direction opposée : en moins d'1 an, la dune a changé de polarité et s'est déplacée d'une trentaine de mètres vers le SW (valeur estimée, figure 5). À l'échelle du court terme, la dynamique des dunes tidales est, par conséquent, fortement contrôlée par les tempêtes. 
Sur la période 1985-1995, la compilation des données de Météo France indique une prédominance des vents de secteur $\mathrm{SW}\left(\mathrm{N} 200^{\circ}\right.$ à $\left.\mathrm{N} 220^{\circ}\right)$ et $\mathrm{NE}\left(\mathrm{N} 20^{\circ}\right.$ à $\mathrm{N} 40^{\circ}$ ). Toutefois, des changements significatifs apparaissent dans le régime des vents (tableau I). Comparativement à la période 1985-1995, les vents de SW sont plus fréquents entre 1992 et $1995(+12,2 \%)$ et moins fréquents entre 1985 et $1992(-4,8 \%)$, alors que ceux de NE sont plus fréquents de 1985 à 1992 $\left(+4,3 \%\right.$ de vents supérieurs à $\left.3 \mathrm{~m} \cdot \mathrm{s}^{-1}\right)$ et moins fréquents de 1992 à $1995(-10,2 \%$ de vents supérieurs à $3 \mathrm{~m} \cdot \mathrm{s}^{-1}$ ). Ces variations du régime des vents expliquent les modifications du schéma de migration des dunes à moyen terme (figure 2). Entre 1985 et 1992, l'augmentation des vents de NE a accentué la migration des dunes du secteur SE vers le SW, et inversé celles des dunes du secteur NW vers le SW. De 1992 à 1995 (figure 2b), les vents de secteur SW ont accéléré le déplacement des dunes du secteur NW vers le NE. Dans le secteur SE, ils ont ralenti la migration des dunes septentrionales vers le SW et ont inversé vers le NE celle des dunes méridionales.

Le vent joue donc un rôle essentiel sur la mobilité des sables dans la zone d'étude. Nos résultats s'accordent avec ceux de Stride [42], qui signale l'effet des courants induits par les tempêtes sur la dynamique sédimentaire des bancs du Norfolk, situés à $200 \mathrm{~km}$ environ au nord de la zone $\mathrm{F}$, à des profondeurs similaires (30-40 m). De même, Johnson [28] et Harris [21, 22] dans le détroit de Torres (Australie), ainsi que Thauront [45] dans le bassin d'Arcachon, suggèrent des modifications saisonnières des courants induites par le vent et conduisant à une inversion de l'asymétrie des grandes dunes.

\section{CONCLUSION}

Le détroit du Pas-de-Calais est un passage marin peu profond où le trafic maritime intense est menacé par l'ampleur des déplacements des grands corps sableux, qui culminent à faible profondeur sous le niveau des basses mers. Dans la partie centrale du détroit, la zone $\mathrm{F}$, définie par le Shom comme «zone à risque pour la navigation ", a été choisie pour l'étude de la dynamique des grandes dunes sableuses. Ces corps sédimentaires, dont les moins profonds sont à $22 \mathrm{~m}$ sous la surface, mettent en danger le transit des bateaux dont le tirant d'eau atteint fréquemment $25 \mathrm{~m}$.

Le champ de dunes est organisé en deux secteurs morphologiquement contrastés : la partie SE est constituée de grandes dunes isolées, la zone NW de plus petites dunes connectées à un banc sableux (South Falls). La frontière morphologique, orientée $\mathrm{N} 45^{\circ}$, est parallèle au grand axe de l'ellipse des courants de marée et coïncide avec une ligne permanente de divergence du transport sédimentaire résiduel.

La morphodynamique des dunes dépend fortement du contexte hydrodynamique. Elle résulte principalement de l'interaction de la marée et des tempêtes. Selon la période de temps considérée, l'une ou l'autre influence prédomine :

Tableau I. Régime des vents sur la période 1985-1995.

Table I. Wind regime between 1985 and 1995.

\begin{tabular}{|c|c|c|c|c|}
\hline Période & & $1985-1995$ & 1985-1992 & 1992-1995 \\
\hline Fréquence : & $\begin{array}{l}\text { des vents de } \mathrm{NE}\left(\mathrm{N} 220^{\circ}-\mathrm{N} 220^{\circ}\right) \\
\text { des vents de } \mathrm{NE}>\text { à } 3 \mathrm{~m} \cdot \mathrm{s}^{-1}\end{array}$ & $\begin{array}{l}8,5 \% \\
6,9 \%\end{array}$ & $\begin{array}{l}8,7 \% \\
7,2 \%\end{array}$ & $\begin{array}{l}8,1 \% \\
6,2 \%\end{array}$ \\
\hline Variation de la fréquence ${ }^{\mathrm{a}}$ : & $\begin{array}{l}\text { des vents de NE } \\
\text { des vents de NE }>3 \text { m.s }{ }^{-1}\end{array}$ & - & $\begin{array}{l}+2,4 \% \\
+4,3 \%\end{array}$ & $\begin{array}{l}-4,7 \% \\
-10,2 \%\end{array}$ \\
\hline $\begin{array}{l}\text { Fréquence des vents de } \mathrm{SW}\left(\mathrm{N} 20^{\circ}-\mathrm{N} 40^{\circ}\right) \\
\text { Fréquence des vents de } \mathrm{SW}>\text { à } 3 \mathrm{~m} \cdot \mathrm{s}^{-1}\end{array}$ & & $\begin{array}{l}14,7 \% \\
12,3 \%\end{array}$ & $\begin{array}{l}14,0 \% \\
12,2 \%\end{array}$ & $\begin{array}{l}16,5 \% \\
12,9 \%\end{array}$ \\
\hline Variation de la fréquence $^{\mathrm{a}}$ : & $\begin{array}{l}\text { des vents de } \mathrm{SW} \\
\text { des vents de } \mathrm{SW}>3 \mathrm{~m} . \mathrm{s}^{-1}\end{array}$ & - & $\begin{array}{l}-4,8 \% \\
-0,9 \%\end{array}$ & $\begin{array}{l}+12,2 \% \\
+4,9 \%\end{array}$ \\
\hline
\end{tabular}

\footnotetext{
a la variation est référencée à la période 1985-1995.
} 
a) La dynamique sédimentaire qui s'établit à long terme (décennie) est contrôlée par les courants de marée et montre une tendance à la rotation horaire. La migration des dunes du SE s'effectue vers le SW sous l'influence du jusant dominant. Les dunes du NW subissent préférentiellement des circulations superposées liées à la proximité du banc sableux du South Falls, et migrent vers le NE.

b) À l'échelle d'une tempête (court terme), les vents perturbent de manière directe et instantanée les caractéristiques du courant sur l'ensemble de la tranche d'eau. Ils peuvent provoquer l'érosion du sommet des dunes, parfois suivie d'une reconstruction avec inversion d'asymétrie.

c) S'ils sont fréquents, les vents modifient à moyen terme (quelques années) les sens de transport sableux résiduels, soit en renforçant, soit en ralentissant, soit encore en inversant l'effet des courants de marée.

La prédiction de la migration des dunes passe par une bonne connaissance des agents hydrodynamiques. Une définition et une classification de ces agents doivent être établies selon deux critères d'influence: leur capacité à mobiliser le sédiment et l'échelle de temps. Le suivi de ces paramètres doit permettre l'établissement d'un modèle régional prédictif de mobilité des corps sédimentaires à court, moyen et long termes, apte à fournir des éléments de compréhension de la dynamique sédimentaire sur les plates-formes épicontinentales. Il doit en résulter une amélioration de la stratégie de surveillance des zones de navigation à risque et une optimisation de la périodicité des levés hydrographiques.

\section{Remerciements}

Ce travail a été réalisé dans le cadre d'un contrat entre le conseil régional du Nord-Pas-de-Calais, le Shom et l'université des sciences et technologies de Lille. Les auteurs remercient B. Tessier et. G. Chapalain pour leurs commentaires pendant la rédaction de l'article. Ils remercient également les rapporteurs dont les commentaires ont permis d'améliorer la qualité de ce manuscrit.

\section{RÉFÉRENCES}

[1] Ashley G.M., Classification of large-scale subaqueous bedforms: a new look at an old problem, J. Sediment. Petrol. 60 (1) (1990) $160-172$.
[2] Belderson R.H., Stride A.H., Bedforms, Tidal current fashioning of a basal bed, Mar. Geol. 4 (4) (1966) 237-257.

[3] Belderson R.H., Kenyon N.H., Stride A.H., Stubbs A.H., Sonographs of the sea floor, Elsevier, 1972, p. 185.

[4] Belderson R.H., Johnson M.A., Kenyon N.H., Bedforms, in: Stride A.H. (Ed.), Offshore Tidal Sand, Processes and Deposits, Chapman \& Hall, Londres, 1982, pp. 27-57.

[5] Berné S., Augustin J.M., Braud F., Chene G., Walker P., Cartographie et interprétation de la dynamique sédimentaire des plates-formes continentales : amélioration de la technique d'observation par sonar latéral, Bull. Soc. géol. Fr. 82 (3) (1986) 437-446.

[6] Berné S., Auffret J.P., Walker P., Internal structure of subtidal sand waves revealed by high-resolution seismic reflection, Sedimentology 35 (1988) 5-20.

[7] Berné S., Allen G., Auffret J.P., Chamley H., Durand J., Weber O., Essai de synthèse sur les dunes hydrauliques géantes tidales actuelles, Bull. Soc. géol. Fr. 6 (1989) 11451160.

[8] Berné S., Bourillet J.F., Durand J., Lericolais G., Ondreas H., Les dunes subtidales géantes de Surtainville (Manche ouest), Bull. des Centres de Recherche Exploration et Production de Elf Aquitaine 13 (2) (1989) 395-415.

[9] Berné S., Castaing P., Le Drezen E., Lericolais G., Morphology, internal structure and reversal of asymmetry of large subtidal dunes in the entrance to Gironde estuary (France), J. Sediment. Petrol. 63 (5) (1993) 780-793.

[10] Boersma J.R., Internal structure of some tidal megaripples on a shoal in the Westerschelde estuary, the Netherlands, Report of a preliminary investigation, Geologie en Mijnbouw 48 (1969) 409-414.

[11] Caston V.N., Linear sand banks in the southern North Sea, Sedimentology 18 (1972) 63-78.

[12] Caston V.N., Stride A.H., Tidal sand movement between some linear sand banks in the North Sea off northeast Norfolk, Mar. Geol. 9 (1970) 38-42.

[13] Clique, P.M., Lepetit, J.P., Catalogue sédimentologique des côtes françaises, côtes de la mer du Nord et de la Manche, de la frontière belge au Mont St-Michel, Électricité de France, Eyrolles, Paris, 1986, 133 p.

[14] Colbeaux J.P., Dupuis C., Robaszynski F., Auffret J.P., Haesaerts P., Sommé J., Le détroit du Pas-de-Calais : un élément dans la tectonique de blocs de l'Europe nord-occidentale, Bull. Inf. Géol. Bass. Paris 17 (4) (1980) 41-54.

[15] Dalrymple R.W., Rhodes N., Estuarine dunes and bars, in: Perillo G.M.E. (Ed.), Geomorphology and Sedimentology of Estuaries, Developments in Sedimentology, Elsevier Science, 1995, pp. 359-422.

[16] De Moor G., Maintenance on the Flemish Banks, in : Henriet J.P., De Moor G. (éd.), The Quaternary and Tertiary Geology of the Soutern Bight, North Sea, Ministry of Economic Affairs and Belgian Geological Survey, 1989, pp. 185216. 
[17] Dyer K.R., Coastal and estuarine sediment dynamics, John Wiley and Sons, 1986, p. 342.

[18] Eisma D., Jansen J.H.F., Vanweering T.G.E., Sea-floor morphology and recent sediment movement in the North Sea, in : Oele E., Schüttenhelm R.T.E., Wiggers A.J. (éd.), The Quaternary History of the North Sea (2), Acta Univ. Ups., Uppsala, 1979, pp. 217-231.

[19] Flemming B.W., Zur Klassifikation subaquatischer, strömungstransversaler Transportkörper, Bochum Geol. U. Geotechn. Arb. 29 (1988) 44-47.

[20] Grochowsky N.T.L., Collins M.B., Boxall S.R., Salomon J.C., Breton M., Lafite R., Sediment transport pathways in the Eastern English Channel, Oceanol. Acta 16 (5-6) (1993) $531-537$.

[21] Harris P.T., Sandwave movement under tidal and wind-driven currents in a shallow marine environment: Adolphus Channel, northeastern Australia, Cont. Shelf Res. 9 (11) (1989) $981-$ 1002.

[22] Harris P.T., Reversal of subtidal dunes assymetries caused by seasonnally reversing wind-driven currents in Torres Strait, northeastern Australia, Cont. Shelf Res. 11 (1991) 655-662.

[23] Heathershaw A.D., Some observations of currents in shallow water during a storm surge, Est. Coast. Shelf Sci. 14 (1982) 635-648.

[24] Herman J.P., Caractérisation des phénomènes hydrodynamiques responsables des mouvements des dunes géantes dans le détroit du Pas-de-Calais, mémoire de DEA (non publié), univ. Lille-1, 1996, 50 p.

[25] Hider A., Observations at Lake Providence, Nov. 1879-Nov. 1880, Progr. Rep. Mississipi river Commission for 1882, 1882.

[26] Houbolt J.J.H.C., Recent sediments in the southern bight of the North sea, Geologie en Mijnbouw 47 (4) (1968) 245-273.

[27] Jelgersma S., Sea-level changes in the North Sea basin, in: Oele E., Schüttenhelm R.T.E., Wiggers A.J. (éd.), The Quaternary History of the North Sea (2), Acta Univ. Ups., Uppsala, 1979, pp. 233-248.

[28] Johnson M.A., Kenyon N.H., Belderson R.H., Stride A.H., Sand transport, in: Stride A.H. (éd.), Offshore tidal sand, processes and deposits, Chapman \& Hall, 1982, pp. 58-94.

[29] Kenyon N.H., Belderson R.H., Stride A.H., Johnson M.A., Offshore tidal sand banks as indicators of net sand transport and as potential deposits, Sp. Pub. Int. Ass. Sedimentologists 5 (1981) 257-268.

[30] L.N.H., Nouvel avant-port de Dunkerque, Mesures en nature, rapp. n³HC042/05 (non publié), laboratoire national d'hydraulique, 1971.

[31] Lüders K., Enstehung und Aufbau von Grossrücken mit Schillbedeckung in flut-bzw, Senckenbergiana 11 (1929) 123142.

[32] McCave I.N., Tidal currents at the North Hinder Lightship,
Southern North Sea: flow directions and turbulence in relation to maintenance of sand banks, Mar. Geol. 31 (1979) 101114.

[33] Perillo G.M.E., Lüdwick J.C., Geomorphology of a sand wave in Lower Chesapeake Bay, Virginia, USA, Geo-Mar. Lett. 4 (1984) 105-112.

[34] Rijn L.C.V., Equivalent roughness of alluvial bed, J. Hydraul. Div., Am. Soc. Civil. Eng. 108 (10) (1982) 1215-1218.

[35] Shom, Courants de marée dans la Manche et sur les côtes françaises de l'Atlantique, service hydrographique et océanographique de la Marine, Paris, 1968, 287 p.

[36] Shom, Atlas des courants de marée, service hydrographique et océanographique de la Marine, Paris, 1988, 16 p.

[37] Smith D.E., Bypassing of sand over sand waves and through a sand wave field in the central region of the southern North Sea, in: De Boer P.L., Van Gelder A., Nio S.D. (éd.), Tide-influenced sedimentary Environments and Facies, D. Reidel Publishing Company, 1988, pp. 39-50.

[38] Smith D.E., Morphological development of the Sandettie South Falls gap: A degeneration ebb dominated tidal passage in the southern North Sea, in : De Boer P.L., Van Gelder A., Nio S.D. (éd.), Tide-influenced sedimentary Environments and Facies, D. Reidel Publishing Company, 1988, pp. 51-64.

[39] Smith J.D., Geomorphology of a sand ridge, J. Geol. 77 (1969) 39-55.

[40] Stride A.H., Current-swept sea floors near the southern half of Great Britain, Q. J. Geol. Soc. London 119 (1963) 175-199.

[41] Stride A.H., Sediment transport by the North Sea, Science (1973) $101-130$.

[42] Stride A.H., Indications of long term episodic suspension transport of sand across the Norfolk Banks, North Sea, Mar. Geol. 79 (1988) 55-64.

[43] Swift D.J.P., Tidal sand ridges and shoal retreat massifs, Mar. Geol. 18 (2) (1975) 105-134.

[44] Terwindt J.H.J., Sand waves in the southern bight of the North Sea, Mar. Geol. 10 (1971) 51-67.

[45] Thauront F., Berné S., Cirac P., Évolution saisonnière des dunes tidales dans le bassin d'Arcachon, France, C.R. Acad. Sci. Paris (IIa) 323 (1996) 411-418.

[46] Van Veen J., Sand waves in the Southern North Sea, Int. Hydro. Rev. 12 (1) (1935) 21-29.

[47] Van Veen J., Onderzoekingen in de Hoofden's Gravenhage, 1936, $252 \mathrm{p}$.

[48] Yalin M.S., Geometrical properties of sand waves, Proc. Am. Soc. Civil Eng. 90 (1964) 105-119.

[49] Zagwijn W.H., Early and Middle Pleistocene coastlines in the southern North Sea basin, in : Oele E., Schüttenhelm R.T.E., Wiggers A.J. (éd.), The Quaternary History of the North Sea, Acta Univ. Ups., Uppsala, 1979, pp. 31-42. 